\title{
Polarized Structure Functions: Proton/Deuteron Measurements in Hall C
}

\author{
Oscar A. Rondon \\ University of Virginia, Charlottesville, VA 22901
}

\begin{abstract}
The study of the nucleon polarized structure functions has matured beyond the inclusive measurements of the past to the investigation of all eight quark distribution functions in the nucleon. Jefferson Lab's Hall C program of polarized structure functions studies started with a measurement of the proton and deuteron spin structure in the resonances at $Q^{2} \sim 1.3[\mathrm{GeV} / \mathrm{c}]^{2}$. This work will be extended for the proton to more than $5[\mathrm{GeV} / \mathrm{c}]^{2}$ for both DIS and the resonances in the upcoming $S A N E$ experiment. SANE will use a novel non-magnetic very large solid angle detector, BETA. Semi-inclusive asymmetries will be measured to determine the flavor composition of the nucleon spin in the recently approved Semi-SANE experiment. The $11 \mathrm{GeV}$ energy upgrade will open new opportunities to study other functions, such as the transversity, Collins and Sievers functions, using vertical polarized targets.
\end{abstract}

Nucleon structure can be described in terms of eight quark distribution functions, three of which are independent of the quark's transverse momentum, and are of leading twist. These three functions are the well measured unpolarized $F_{1}$ and polarized $g_{1}$ structure functions (SF's), and the yet to be determined transversity $h_{1}$, or $\delta$. The five transverse momentum dependent SF's include the polarized transverse SF $g_{T}=$ $g_{1}+g_{2}$, the Collins $h_{T}^{\perp}$ fragmentation function and Sievers $f_{1 T}^{\perp}$ distribution function. Only three of these distribution functions can be explored in inclusive inelastic leptonnucleon scattering $\left(F_{1}, g_{1}\right.$ and $\left.g_{2}\right)$. The others require the measurement of semi-inclusive polarized asymmetries, with one hadron detected in coincidence with the lepton.

Jefferson Lab's Hall C program of polarized nucleon structure measurements started in the year 2002 with an inclusive measurement (E-01-006) of $g_{1}$ and $g_{2}$ in the resonances for protons and deuterons at intermediate four-momentum transfer $Q^{2} \sim 1.3$ $[\mathrm{GeV} / \mathrm{c}]^{2}$. The Spin Asymmetries on the Nucleon Experiment(SANE) will extend these inclusive measurements for the proton to $Q^{2} \sim 6[\mathrm{GeV} / \mathrm{c}]^{2}$, in the Bjorken $x$ range of 0.3 to 0.8 . Semi-inclusive polarized asymmetries to study the quark flavor composition of the nucleon spin will be measured in another experiment which will share with $S A N E$ the Big Electron Telescope Array (BETA) to detect electrons.

\section{TJNAF E-01-006 - RESONANCES SPIN STRUCTURE (RSS)}

The leading twist spin structure functions (SSF's) $g_{1}$ and $g_{2}$, and their related spin asymmetries $A_{1}$ and $A_{2}$ require the measurement of two asymmetries for different relative orientations of the target spin and the lepton beam helicity. The usual approach is to measure the asymmetries with the nucleon spin parallel $\left(A_{\|}\right)$and perpendicular $\left(A_{\perp}\right)$ 
to the beam helicity. There is a significant scarcity of $A_{\perp}$ measurements for much of the $Q^{2}-x$ kinematic plane for $x>\sim 0.5$, which is the focus of this workshop, in particular for $Q^{2}$ below $\sim 6[\mathrm{GeV} / \mathrm{c}]^{2}$.

Much of the high $x$ region for $Q^{2}$ up to $\sim 10[\mathrm{GeV} / \mathrm{c}]^{2}$ corresponds to final state invariant mass $W \leq 2 \mathrm{GeV}$, which is dominated by the nucleon resonances. The first Hall C experiment on the SSF's, $R S S^{1}$, concentrated on a precision measurement of proton and deuteron $A_{1}$ and $A_{2}$ in the resonances. The main goals of this experiment are to study the $W$ dependence of the SSF's, look for the onset of polarized local duality, and explore the contribution of twist-3 processes to $g_{2}$.

$R S S$ measured $A_{\|}$and $A_{\perp}$ for protons and deuterons by scattering $5.76 \mathrm{GeV}$ polarized electrons accelerated with CEBAF on polarized frozen ammonia $\left(\mathrm{NH}_{3}\right)$ and deuterated ammonia $\left(\mathrm{ND}_{3}\right)$ targets. The scattered electrons were detected in the High Momentum Spectrometer-HMS parked at $13.15^{\circ}$. Two settings of the HMS central momentum were used, 4.73 and $4.09 \mathrm{GeV} / \mathrm{c}$. The kinematic range covered with these parameters extends from $0.6 \mathrm{GeV}$ to $2 \mathrm{GeV}$ in $W$ at $Q^{2} \approx 1.3 \pm 0.3$.

The measured asymmetries are obtained from the ratio of the difference over the sum of charge normalized event counts for opposite beam helicities, $N_{L}, N_{R}$. This ratio is normalized by the beam polarization $P_{b}$, target polarization $P_{t}$ and dilution $f$ of the polarized nuclei by unpolarized nucleons in the target (nitrogen, liquid He, etc.)

$$
A_{\|, \perp}=\frac{1}{C_{N} f_{R C}}\left(\frac{N_{L}-N_{R}}{N_{L}+N_{R}} \frac{1}{f P_{b} P_{t}}-C_{D}\right)+A_{R C} .
$$

The asymmetry is also corrected for the contributions of radiative effects, $f_{R C}, A_{R C}$ and the small nitrogen polarization $C_{N}, C_{D}$.

The usable beam current was limited by the cooling power of the polarized target to $200 \mathrm{nA}$ or less. In addition, the beam needed to be rastered over a $2 \mathrm{~cm}$ diameter circle to spread the damage induced by the beam radiation and reduce the resulting rapid local polarization loss. The beam polarization was measured with the Hall's Møller spectrometer. For the perpendicular asymmetry part of the experiment the polarization was $66 \% \pm 0.5 \%$ and $71 \% \pm 0.5 \%$ for the parallel data.

The out of plane offset due to the beam rastering requires a special treatment in the event reconstruction. A special set of spectrometer optics reconstruction matrix elements was used to correct for the out of plane offset in a consistent way in the data analyzer and the Montecarlo simulation.

The polarized target operated at $5 \mathrm{~T}$ and $1 \mathrm{~K}$. Dynamic nuclear polarization of the ammonia by microwave irradiation produced in-beam proton polarizations of $70 \%$ and deuteron polarizations of $20 \%$. The polarization was measured continuously during the experiment by the NMR technique. The intense magnetic field of the target produces large deflections in the trajectories of the scattered electrons, especially in the perpendicular configuration. These deflections need to be known very accurately to obtain the

${ }^{1}$ RSS Collaboration (JLab E-01-006): U. Basel, Florida International U., Hampton U., U. Massachusetts, U. Maryland, Mississippi S. U., North Carolina A\&T U., U. of N. C. at Wilmington, Norfolk State U., Old Dominion U., S. U. New Orleans, U. of Tel-Aviv, TJNAF, U. of Virginia, Virginia P. I. \& S. U., Yerevan Physics I. Spokespersons: O. A. Rondon (Virginia) and M. Jones (TJNAF). 

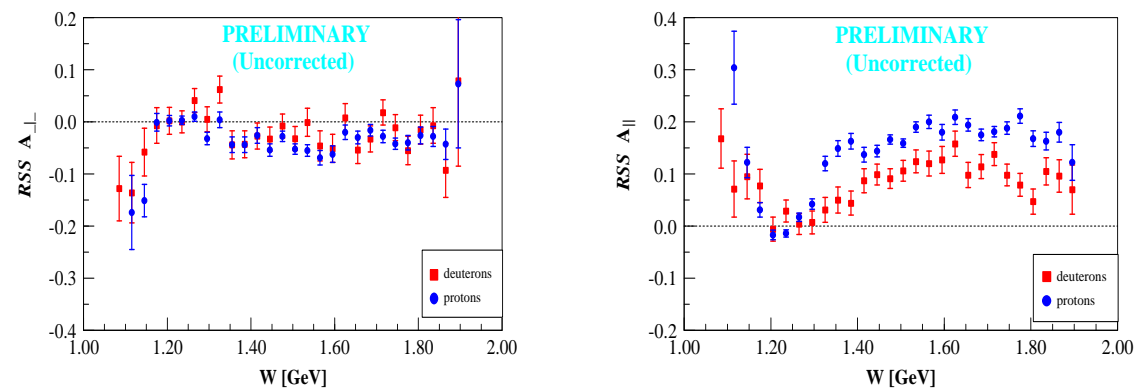

FIGURE 1. Preliminary measured asymmetry for the perpendicular confi guration (left) and the parallel one (right) for protons (circles) and deuterons (squares), without corrections (see text for details.

correct kinematics for the reconstructed events. RSS is unique in that it is the only experiment to date to have measured high resolution perpendicular asymmetries on solid polarized targets in the $e-p$ elastic and nucleon resonances regions.

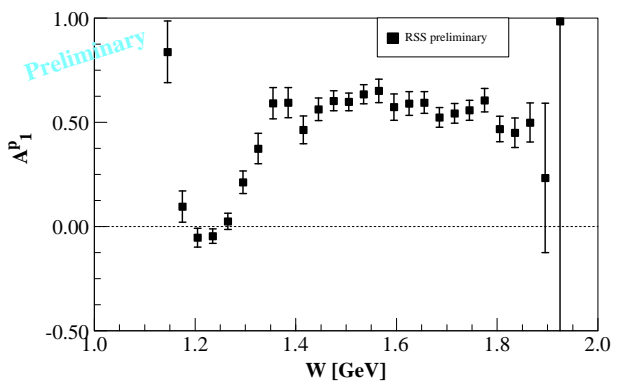

FIGURE 2. Preliminary proton $A_{1}$.

The dilution of the asymmetries by the unpolarized nucleons is calculated using a Montecarlo simulation of the yields for each material. Model Born cross sections from the $Q F S$ [1] software package were combined with parameterizations [2] of single-arm proton and deuteron electro-nuclear cross sections measured in Hall $\mathrm{C}$. The fraction by volume of ammonia in the target cells is specific for every cell load, ranging from about $50 \%$ to about $60 \%$. A nominal $50 \%$ value was used for the preliminary dilution factors.

The uncorrected preliminary $A_{\perp}$ and $A_{\|}$are shown in Figure 1. These asymmetries are based on the first pass version of the data analysis and preliminary dilution factors. Corrections for individual cells' packing fractions, nitrogen asymmetry and radiative 
effects have not been applied. Since $A_{1}$ is mainly dependent on $A_{\|}$, which is not very sensitive to the details of the target field (the angle between the field and the scattered electrons is only $13^{\circ}$ ), we have used the preliminary $A_{\|}$and $A_{\perp}$ to obtain preliminary, uncorrected results for the proton $A_{1}$, which are shown in Figure 2.

\section{TJNAF E-03-009 - SPIN ASYMMETRIES ON THE NUCLEON EXPERIMENT - SANE}

$S A N E^{2}$ will extend the measurements of the preceding experiment into the $Q^{2}$ range of $2.5[\mathrm{GeV} / \mathrm{c}]^{2}$ to $6.5[\mathrm{Gev} / \mathrm{c}]^{2}$ for proton targets, in the DIS and resonances, for $0.3 \leq$ $x \leq 0.8$. The goals of $S A N E$ are to measure both $g_{2}$ and $A_{1}$ over a broad range of $x$ and $Q^{2}$ to study their $x$ dependence at constant $Q^{2}$, and $Q^{2}$ dependence at fixed $x$, in order to compute the moments of the SSF's. The third moments are related to the $d_{2}$ twist-3 quark matrix element $d_{2}=\int_{0}^{1} x^{2}\left(2 g_{1}+3 g_{2}\right) d x$, which can be calculated in Lattice QCD. Polarized or spin local duality will be tested down to the second resonance, too.

Polarized electron beams of 6 and $4.8 \mathrm{GeV}$ energy will be scattered on polarized solid ammonia targets. The proton spins will be aligned parallel to the beam and an $80^{\circ}$ angle to measure parallel and near-perpendicular asymmetries, for a model independent separation of $g_{1}$ and $g_{2}$.

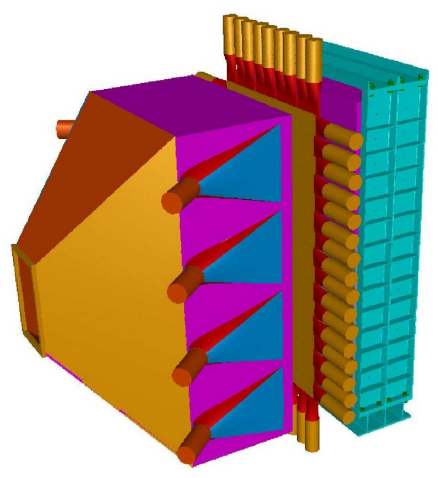

FIGURE 3. Artist view of BETA. The sideways pyramid shaped object at left is the gas Cherenkov, the stack of rectangular blocks on the right is BigCal. The tracking hodoscope is sandwiched in between.

To compensate for the decrease in cross section with increasing $Q^{2}$ and $x, S A N E$ is building a new type of non-magnetic large solid angle detector for electrons, the Big Electron Telescope Array (BETA). This detector will be aligned with its axis at $40^{\circ}$ to the left of the beam. The HMS will be aligned at $40^{\circ}$ to the right of the beam to measure the small asymmetry for the the positrons produced in pair-symmetric processes, which

\footnotetext{
2 TJNAF E03-109: U. Basel, Florida International U., Hampton U., Norfolk State U., IHEP Protvino, U. of Regina, Rensselaer P. I., Seoul U., Temple U, TJNAF, U. of Virginia, C. of William \& Mary, Yerevan Physics I. Spokespersons: O. Rondon (Virginia), Z-E. Meziani (Temple), S. Choi (Seoul).
} 
contaminate the electro-nuclear events, especially at low momenta. BETA consists of three main subsystems:

BigCal is a 1700-element lead glass calorimeter being built by the BigCal collaboration for the high $Q^{2}$ measurement of the proton elastic form factor ratio $G_{E}^{p} / G_{M}^{p}$ [3]. It is the main component of the detector package, covering a 194 msr solid angle (after cuts). Its energy resolution is $d E / E \simeq 5 \% / \sqrt{E}$. The uniformity of the response of all the elements will be monitored by a Lucite plate excited by laser light [4].

Gas Cherenkov will be the main particle identification device, with expected 1000:1 pion rejection capability in combination with BigCal. The Cherenkov matches BigCal's solid angle. It is being built by the Temple University group [5].

Tracking Hodoscope based on Cherenkov light emission in transparent solids (Lucite or quartz - choice of material under study). The hodoscope will add an extra level of background rejection by tracking events in the calorimeter back to the target.

Figure 3 is an artist's view of BETA. The final design and element configuration is being actively studied at the time of this report.
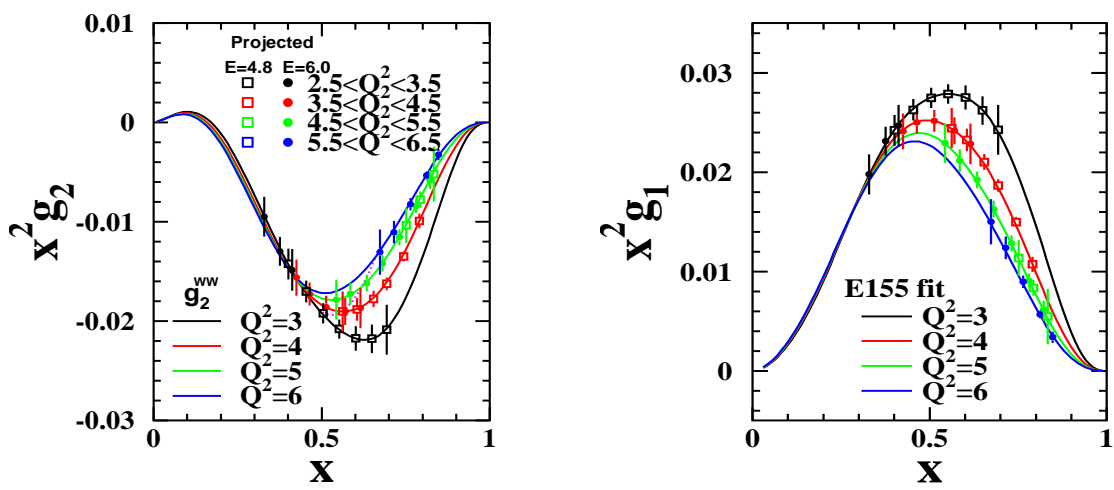

FIGURE 4. Expected results for the integrands of the third moments of $g_{2}$ (left) and $g_{1}$ (right). Both the $x$ dependence at constant $Q^{2}$ and the $Q^{2}$ dependence at fi xed $x$ will be tested.

Examples of the expected $S A N E$ results for $g_{2}$ and $g_{1}$ are shown in Figure 4. With $S A N E$ 's results, the current statistical error on $d_{2}$ will be cut in half. More examples for $g_{2}, A_{1}$ and tests of polarized local duality, not shown here due to space limitations, can be found at SANE's Web site http: / /www. jlab.org/ rondon/sane/.

\section{TJNAF E-04-013 - FLAVOR DECOMPOSITION OF NUCLEON SPIN STRUCTURE}

The contributions of each quark flavor to the nucleon spin can only be measured in electro-nuclear scattering by tagging recoil hadrons (pions, kaons), that are detected 
in coincidence with the lepton. Experiment $04-113^{3}$ will measure proton and deuteron semi-inclusive spin asymmetries in polarized DIS reactions $\vec{p}\left(\vec{e}, e^{\prime} h\right)$ and $\vec{d}\left(\vec{e}, e^{\prime} h\right)$, where $h$ symbolizes $\pi^{ \pm}, K^{ \pm}$. The measurement will extend over the ranges $1.2[\mathrm{GeV} / \mathrm{c}]^{2} \leq Q^{2} \leq$ $3.2[\mathrm{GeV} / \mathrm{c}]^{2}$ and $0.12 \leq x \leq 0.43$, for hadrons carrying a fraction $0.5 \leq z\left(=E_{h} / \mathrm{v}\right) \leq 0.7$ of the energy transfer $v$. Polarized electrons of $6 \mathrm{GeV}$ beam energy will be scattered from longitudinally polarized protons in $\mathrm{NH}_{3}$ and deuterons in $\mathrm{LiD}$. The scattered electrons will be detected in $B E T A$, parked at $30^{\circ}$, in coincidence with hadrons detected in the HMS aligned at $10.8^{\circ}$.

The valence quark helicities will be obtained using the LO and NLO Leader-Christova method [6], in which the difference of asymmetries for both signs of the hadron charges

$A_{1 p, d}^{h^{+}-h^{-}}$is combined with inclusive results on the SSF's to get the polarized light sea flavor asymmetry. In addition, by forming the semi-inclusive asymmetries at fixed $z$ in terms of fragmentation function (FF) ratios, the five quark flavor helicity distributions can be obtained from a global fit to all fixed $z$ data, using known parton distribution functions (PDF). The combined asymmetry sums $A_{1 p, d}^{h^{+}+h^{-}}$and the inclusive $A_{1}^{p, d}$ data can be compared to test factorization of the asymmetries into PDF's times FF's.

The experiment is expected to improve the current errors on flavor helicities by factors of 3 or better, and to clearly establish whether the polarized light sea is asymmetric. The wealth of results expected from this experiment cannot be properly presented in the limited space available. For extensive detailed information the reader is referred to the proposal document [7].

\section{ACKNOWLEDGMENTS}

This work was supported by Department of Energy contract DE-FG02-96ER40950 and by the Institute of Nuclear and Particle Physics of the University of Virginia.

\section{REFERENCES}

1. Lightbody, J., and O'Connell, J., Computers in Physics, May/June 1988, pp. 57-64 (1988).

2. Keppel, C., private communication (2003).

3. Brash, E., Jones, M., Perdrisat, C., and Punjabi, V., Measurement of $G_{E}^{p} / G_{M}^{p}$ to $Q^{2}=9 \mathrm{GeV}^{2}$ via Recoil Polarization, Tech. rep., TJNAF Proposal E-04-108 (2004).

4. Frlez, E. (2004), URL http: //www.jlab.org/ choi/Sane/Frlez.pdf.

5. Meziani, Z.-E. (2004), URL http://www.jlab.org/ rondon/sane/mtgl/cherenkov . pdf.

6. Christova, E., and Leader, E., Nucl. Phys., B607, 369-390 (2001).

7. Jiang, X., Flavor Decomposition of Nucleon Spin Structure, Tech. rep., TJNAF Proposal E-04-113 (2004), URL http://www.jlab.org/exp_prog/generated/apphallc.html.

\footnotetext{
3 TJNAF E03-109: Argonne National Lab, Duke U., Florida International U., Hampton U., U. Kentucky, U. Maryland, U. Massachusetts, Norfolk State U., Old Dominion U., IHEP Protvino, U. of Regina, Rensselaer P. I., Rutgers U., Seoul U., Temple U, TJNAF, U. of Virginia, C. of William \& Mary, Yerevan Physics I. Spokespersons: X. Jiang (Rutgers), P. Bosted (TJNAF), D. Day (Virginia), M. Jones (TJNAF).
} 